\title{
Non-linear transport and topology of dielectric breakdown
}

\author{
J.C. Maan ${ }^{\mathrm{a}, *}$, B. Willing ${ }^{\mathrm{b}}$, P. Uhlig \\ ${ }^{a}$ High Field Magnet Laboratory, Research Institute for Materials, University of Nijmegen, NL-6525 ED Nijmegen, The Netherlands \\ ${ }^{\mathrm{b}}$ High Magnetic Field Laboratory, Max Planck Institut für Festkörperforschung, I66X-F 38042 Grenoble 9, France
}

\begin{abstract}
Isolators subjected to a sufficiently high electric field become conductors due to electrical breakdown. This transformation into conductor is an example of extremely non-linear (non-ohmic) conductivity. A consequence of this non-linearity is that the topology of the electric field and current distribution is not determined anymore by the externally applied voltages but rather by the local, time dependent, conductivities. Experiments of breakdown in air (Sparks) and in semi-insulating GaAs are discussed as examples of, respectively, current filaments and of electrical domain formation. For current filaments, a magnetic field is shown to lead to a "fractal-like" pattern with a "dimension" determined by the external field.
\end{abstract}

\section{Introduction}

The physics of non-linear phenomena is a central modern research area. The reasons for this interest comes from the wealth of new and unexpected physical phenomena as soon as the simple linear approximations are abandoned. Furthermore, many systems in the real world, as opposed to the ideal systems usually studied, show a behaviour which can only be understood from non-linear dynamics. Examples are non-linearities in chemical reactions, heat flow (Rayleigh-Bénard instabilities), hydrodynamics (Couette instabilities) and nonlinear optics $[1,2]$. Breakdown of dielectric media (air, gases but also semi-insulating semiconductors) belongs to this same class of non-linear phenomena [3].

All non-linear phenomena have in common the spontaneous breaking of space and time symmetry which leads to a characteristic spatiotemporal pattern formation. The beauty of the patterns which spontaneously appear when driving the system in the non-linear regime upon the smooth increase of an external parameter has

\footnotetext{
* Corresponding author.
}

certainly contributed to the fascination of many physicists for the subject.

In this paper we want to review two experiments concerning pattern formation caused by the strongly non-linear conductivity at dielectric breakdown. In one example we investigate the topology of discharge patterns in electrical sparks in a magnetic field and in the other the shape of electric field domains in a semi-insulating semiconductor (SI-GaAs) subjected to a high electric field. These two examples illustrate the two typical patterns encountered in dielectric breakdown, namely the formation of current filaments and of electrical domains.

At the origin of these two different topologies lies the different shape of the current density $(j)$ as function of electric field $(E)$ characteristic, which may be either Nshaped (NNDC) or S-shaped (SNDC), both exhibiting negative differential conductivity (NDC) (see Fig. 1). As indicated in the figure, for the NNDC, a line at constant current density intersects the $j-E$ characteristic of three different values of the electric field in the non-linear region. Similarly for the SNDC a line at constant electric field intersects the $j-E$ curves at three different values of the current density $j$. Therefore, a system with an NNDC 


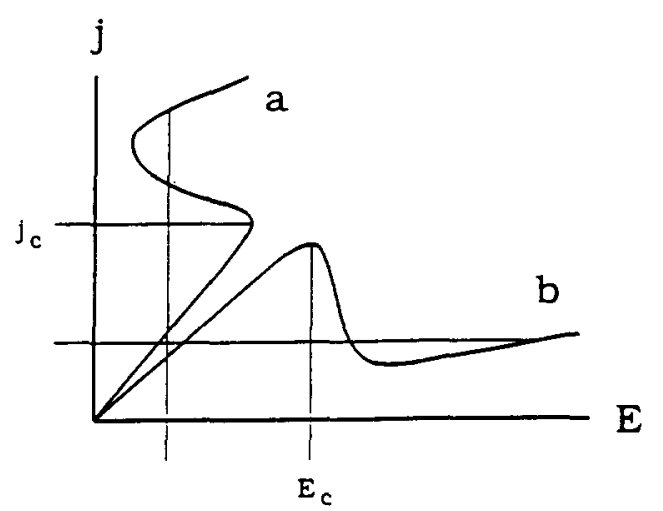

Fig. 1. Schematic representation of typical S-shaped (a) and $\mathrm{N}$-shaped (b) electrical non-linearities, leading to current filaments above $j_{\mathrm{c}}$ for $\mathrm{S}$-shaped and to domains for $\mathrm{N}$-shaped characteristics above $E_{\mathrm{c}}$.

will subdivide in regions with different electric fields at the same current density (domain formation), and one with a SNDC in filaments with a different current density at the same electric field.

\section{Pattern formation of electrical discharges in air}

Surface discharge patterns lead to intricate and beautiful figures. These figures can be generated in a point-toplane configuration, where a high voltage pulse is applied to a point electrode placed on top of a thin insulating dielectric film on a conducting counter electrode. Such patterns were originally studied by Lichtenberg as early as 1778 [4] and are therefore often referred to as Lichtenberg figures. A very convenient method to make them visible is to use a photographic film as the dielectric medium and to create the discharge in a dark environment. The breakdown, caused by ionisation of the gaseous medium, is accompanied by the emission of light, which leaves directly a replica of the figure on the photographic plate. The basic physical mechanism underlying the pattern is that in a sufficiently high electric field the small number of free electrons $\left(10^{4} \mathrm{~m}^{-3}\right.$ in air at ambient temperature and pressure for instance) gain enough kinetic energy to create secondary electrons through ionization of neutral atoms and molecules. The shape of the pattern depends on a number of parameters, such as the type of gas used, the shape of the electrode, the polarity and the magnitude of the voltage of the point electrode, the thickness and nature of the dielectric plate, etc. [5].

In the point-to-plane geometry the potential distribution at low voltages in the vicinity of the point and along the plane of the dielectric follows basically a $1 / r$ law with $r$ the distance from the point. Secondary electrons are created near the point and are accelerated by the field. Charged ions (which are much slower) are left behind and the accumulated space charge tends to screen the applied field. When the voltage is not too high a quasi-stationary state between ionization by the field and recombination occurs in the vicinity of the point. This "glow discharge" or "corona" (well-known as the so-called Elm's fire) is usually well-evolved for positive DC voltages and only seen with high pulsed excitation for negative voltages.

As the voltage is increased, ionization in the glow discharge region increases which leads to a radially extending filamentary structure. Finally upon further increase of the voltage due to the ionization and carrier heating, some of these filaments become so highly conducting that the voltage drop along the conducting path becomes negligible and the point potential is almost fully transferred to the tip of the conducting channel. The glow discharge phase then starts again at the tips of all channels, and this process leads eventually to the pattern [6]. The process is of stochastic nature and leads to a disappearance of the original circular symmetry.

Fig. 2 shows typical patterns for $13.6 \mathrm{kV}$ pulses of $500 \mathrm{~ns}$ in air for a positive and a negative potential of the tip. For the positive tip a highly branched structure develops whereas long, more or less straight, radial spikes are formed for a negative tip. This difference in shape is a direct consequence of the fact that for positive voltages the electrons are accelerated in an increasing electric field towards the tip while for negative potentials they move outwards to lower electric field region. Therefore, the necessary length to obtain sufficient energy to ionize secondary electrons is much shorter for positive than for negative potentials, which makes that in the latter case the radially oriented guiding field is more important.

Niemeyer et al. [7] have shown that positive discharges patterns may be described by fractal behaviour. The term fractal means that the structure of an object appears to be self-similar on all length scales, i.e. the structure of a "magnified" part of the object looks the same as the object itself. The continuous bifurcation at a finer and finer scale of the positive discharge shown in Fig. 1 is reminescent of this fractal behaviour [8]. It is important to note that the bifurcating pattern never leads to a crossing of the various branches. This occurs because the already formed channels are at almost the same potential as the point, therefore the potential differences between several branches is almost zero, whereas each branch is at a high potential with respect to the nonionized medium in between. As two branches approach each other the electric field between them diminishes, making it more and more probable to change the direction of propagation since the highest electric field (and 


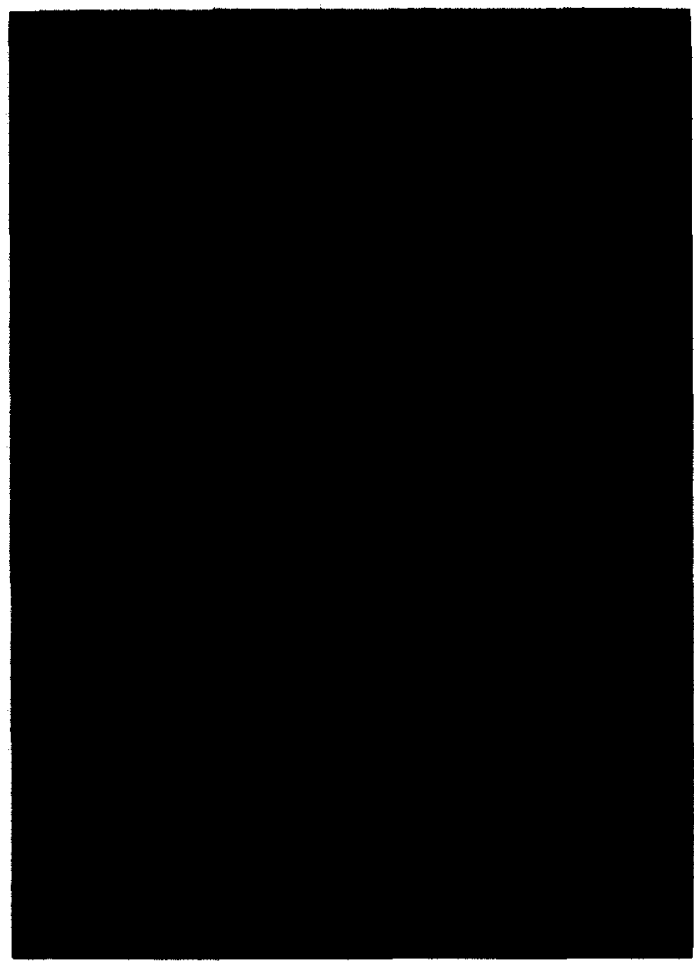

Fig. 2. Comparison between surface discharges with a positive point potential (top) and a negative point potential (bottom) under otherwise equal experimental conditions $(500 \mathrm{~ns}, 13.6 \mathrm{kV}$ pulses in air).

thus the strongest ionization) will now occur in another direction. For a negative discharge this bifurcating and fractal behaviour is clearly not observed in this example. The longer lasting influence of the guiding field makes that the "spokes" of the spark become far apart and once they are far from the tip they continue to grow radially.

This breakdown in air is a typical example for current filament formation. Due to the liberation of secondary electrons the conductivity increases with increasing electric field giving rise to the S-shape $j-E$ characteristic discussed in the introduction. The distinct branches of the spark can directly be interpreted as the current filaments, formed as a consequence of this non-linear conductivity.

\section{The effect of a magnetic field on negative discharges in air}

The negative discharge pattern changes drastically when a magnetic field is applied perpendicular to the plane of the surface discharge. Fig. 3 shows the evolution of the discharge pattern at four different magnetic fields.
The radially propagating electrons experience the Lorentz force, perpendicular to their propagation direction under the influence of a magnetic field and this leads to curved paths of the spark. In the vicinity of the electrode the electric field is radially oriented, therefore the force on the electrons has one radial component and one component perpendicular to the propagation direction. Therefore, the path of the electrons has a constant angle between the propagation direction and radial direction, which is referred to as the logarithmic spiral; well-known from the Corbino geometry in measurements of the magnetoresistance. This spiral path is visible in Fig. 3(b)-(d) near the centre of the spark.

At larger distances from the centre, the applied radial field becomes irrelevant because the potential distribution is now determined by the already formed conducting channels in the discharge (the dark branches in the figure) which are almost at the same potential as the point. The tips of these conducting channels start to ionize the gap in their vicinity and propagate in this medium [6].

The transition from the importance of the radial field near the centre to the field distribution of the already formed tip of channels is directly visible as a change in the shape of the spark paths from a logarithmic spiral near the centre to circular trajectories further outside. The fact that the original radial field becomes irrelevant can directly be seen from Fig. 3(b) where some branches propagate even towards the central electrode and thus against the radial field [9].

An important consequence of the circular shape of the paths is that different branches which at zero field become further apart as they extend outward, now approach each other. For the same reason as discussed with the positive sparks, bifurcation will occur when branches approach each other. This is why the patterns in Fig. 3 show an increasing complexity and fill the two-dimensional space more evenly with increasing magnetic field. A magnetic field can thus be used as an external parameter to vary this complexity.

At the highest magnetic field three "generations" of curved paths can be distinguished, each with its own, to the outside of the figure decreasing, radius of curvature. It is tempting to interpret these patterns as fractal objects showing some form of similarity at different length scales. Such an analysis should of course be treated with care since the finite extension of our resolved individual branches limit severely the range of length scales at which we can study our figure. Nevertheless we will use the mathematical models to describe fractal growth; bearing its deficiencies in mind.

For a truely fractal figure of arborescent nature the following relation holds [10]:

$n(r)=r^{d_{f}-1}$. 


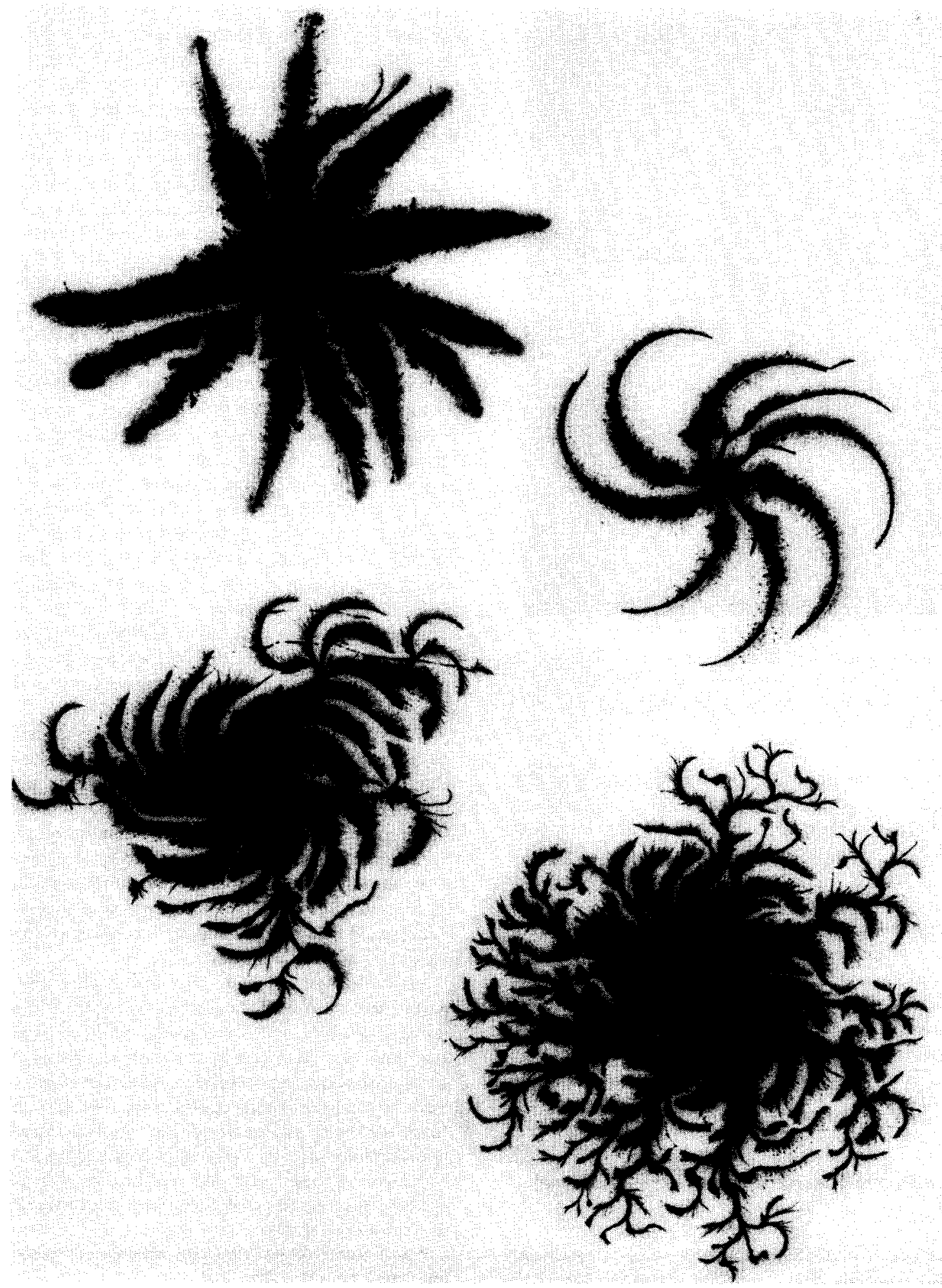

Fig. 3. Negative leader discharges in air at atmospheric measure and temperatures for $B=0(\mathrm{a}), B=5 \mathrm{~T}$ (b), $B=9 \mathrm{~T}(\mathrm{c}), B=12 \mathrm{~T}$ (d). 

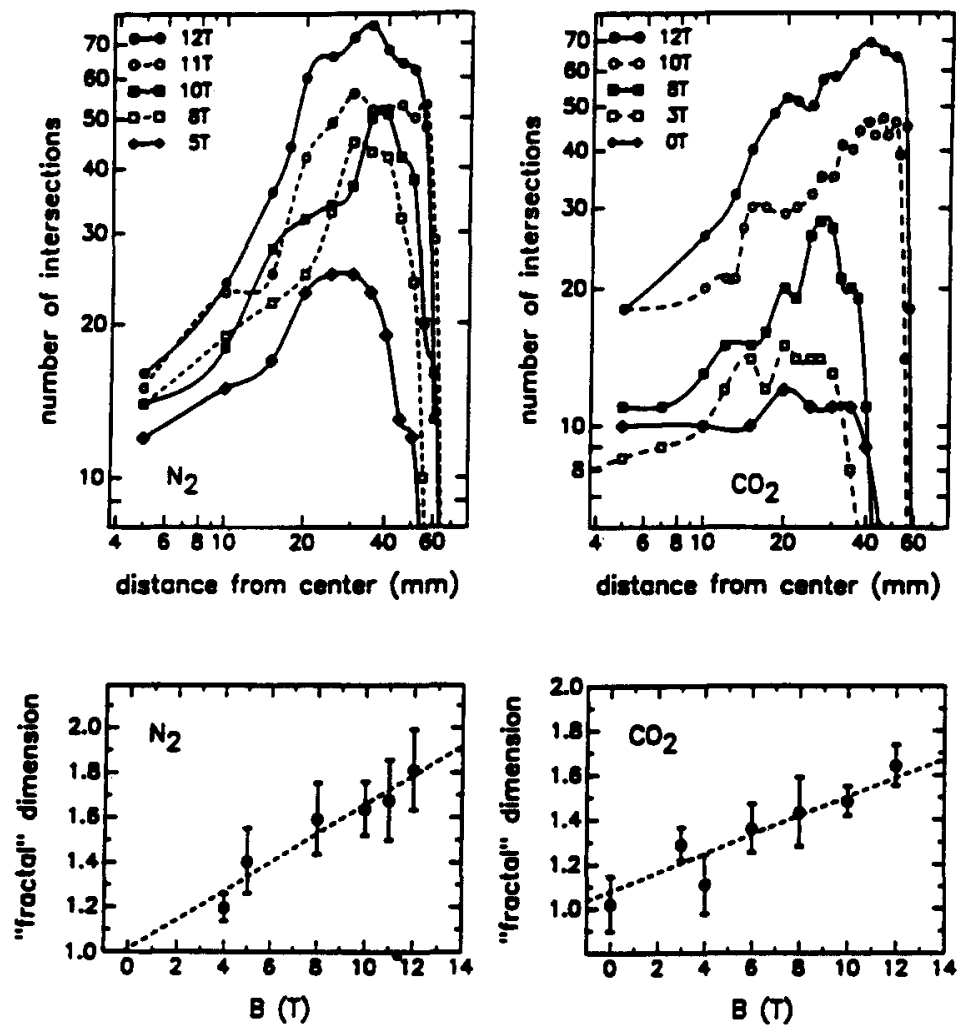

Fig. 4. (a) Radial density of discharge branches for (individual) discharges in $\mathrm{N}_{2}$ and $\mathrm{CO}_{2}$, respectively. (b) "Dimension" $d_{\mathrm{f}}$ deduced from a linear regression to the data in (a).

Here $n(r)$ is the number of intersections of branches with a circle at a distance $r$ from the centre and $d_{\mathrm{f}}$ the "fractal dimension". For an object which grows occupying space fully $d_{\mathrm{f}}=3$ and $d_{\mathrm{f}}=2$ for three or two dimensions, and as expected $n(r)$ increases as $r^{2}$ or $r$, respectively. For figures occupying space more sparsely, $d_{\mathrm{f}}$ will be smaller than the dimension of the space.

For the characterization of our discharge patterns we used (e.g. 1) and counted the intersections as a function of the radial distance, taking care to count even the tiniest branches. If the power law (e.g. 1) holds, the data should fall on a $\log -\log$ plot of $n(r)$ with a characteristic slope given by $d_{\mathrm{f}}$. The results are shown in Fig. 4 for different magnetic field for negative discharge patterns in $\mathrm{CO}_{2}$ and $\mathrm{N}_{2}$. Although, there is as expected, a significant scattering in the data, there is a clear trend of an increase of $n(r)$ at as a function of magnetic field, parametrizing the increasing complexity of the figure. A least mean squares fit of the slopes in the top figures as a function of field is shown in the lower part. The "fractal dimension" is seen to increase linearly, from 1 at $0 \mathrm{~T}$ to $\simeq 1.70$ at $12 \mathrm{~T}$. These limiting values are quite reasonable since at low fields the channels are progressing linearly and their dimension is expected to be 1 while at high fields the value of $\simeq 1.7$ is close to 1.75 found by Niemeyer et al. [7] for positive figures in $\mathbf{S F}_{6}$ at zero field both experimentally and theoretically.

This analysis clearly shows that a magnetic field can be used to vary the topology of a discharge patterns in a very direct manner.

\section{Visualization of dielectric breakdown in semi-insulating GaAs}

Dielectric breakdown is not unique for gaseous media but can occur in any isolator providing the field is high enough. Semi-insulating (SI)GaAs, as used for substrate wafers, also shows a strongly non-linear behaviour. Because the energies of the relevant states in this system is much lower $(\simeq 0.7 \mathrm{eV})$ compared to the ionization energies involved in gaseous media $(\simeq 15 \mathrm{eV})$, the corresponding voltages are much lower. However, the simple visualization technique of photographic plates cannot be 
used to study directly the shape of the breakdown pattern.

SI-GaAs has an $\mathrm{N}$-shaped $j-E$ characteristic, therefore electrical domain formation is expected, as opposed to current filament formation discussed in connection with breakdown in air. In order to observe directly the local, time-dependent, electric field distribution at electrical breakdown we used a recently developed electrooptical technique. A plate of electrooptically active material, coated on one side, with a dielectric mirror and, on the other, with a transparent electrode is placed with the dielectric coating facing down on top of the sample. The
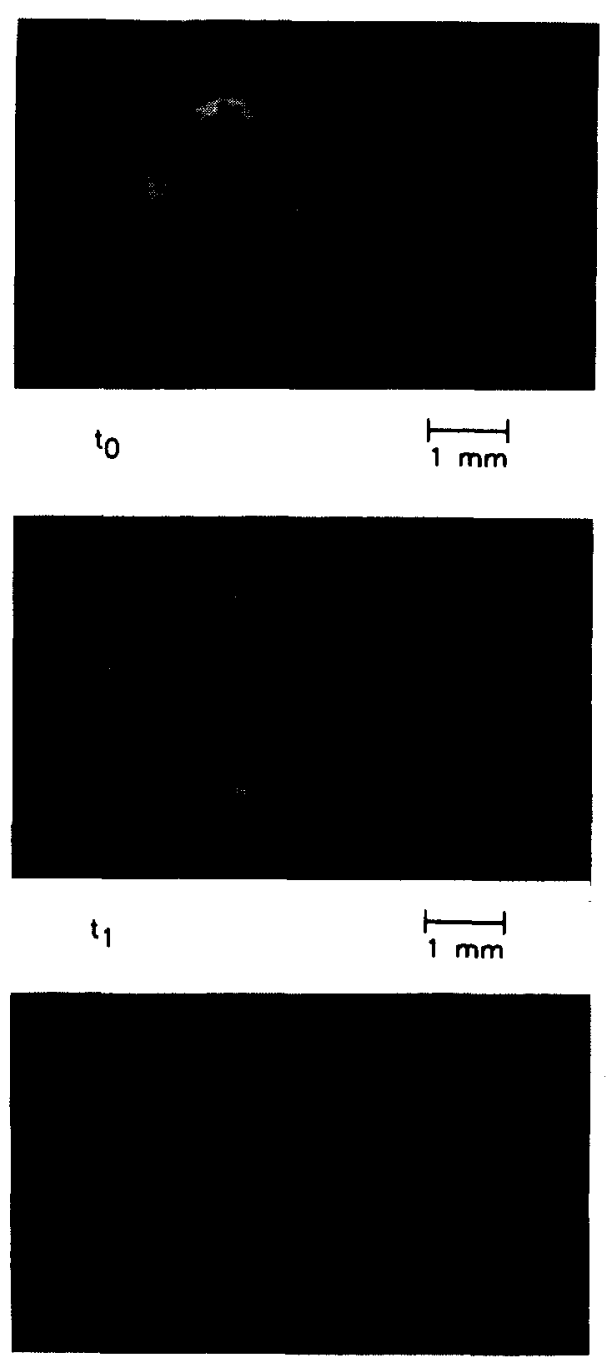

$t_{2}$

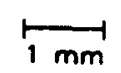

image of the sample between crossed polarisers, using monochromatic light is observed with a video camera. In this way, we obtain on the screen a map of the sample where the local light intensity, due to the change in polarization of the electrooptic crystal, is exclusively determined by the local electric field in the sample. Beyond the direct visualization of the domain topology and propagation, a subsequent data analysis of the image provides a quantitative measurement of the local, timedependent, electric field distribution of the sample.

In the experiment a semi-insulating sample of $\mathrm{GaAs}$ is biased with a sufficiently high voltage to drive the system
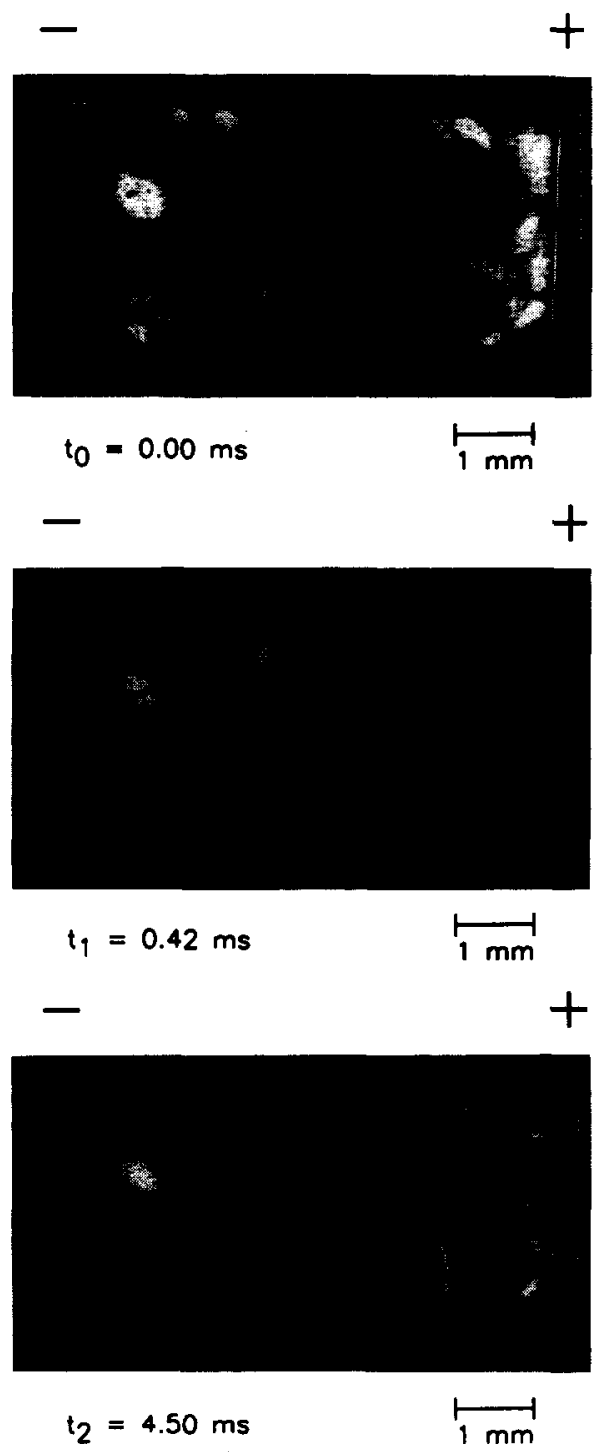

Fig. 5. The shape and the position of an electrical domain formed during breakdown of SI-GaAs for a rectangular sample (left) and a sample with two point-like electrodes right at different time intervals 1, 2 and 3 . 
into the non-linear conductance regime. The entering of this regime is detected by the appearance of spontaneous current oscillations at constant voltage bias. These oscillations are caused by the formation of high electric field domains, which propagate through the sample, leading to a time-dependent overall conductivity. At the origin of the domain formation lies the negative differential conductivity of SI GaAs. Due to trapping of fast carriers on deep traps (the EL2 defect in this case $[3,11]$ ) the carrier density decreases with increasing electric field leading to an $\mathrm{N}$-shaped $j-E$ characteristic as shown in Fig. 1. Negative differential conductivity lies also at the origin of the well-known Gunn effect [12], but the mechanism is in that case an electric field induced reduction in the mobility due to transfer of electrons to a conduction band state with a much higher mass. A consequence of this difference is that the domain in SI GaAs propagate much slower leading to very low frequency $(0.1-100 \mathrm{~Hz}$ as opposed to several $\mathrm{GHz}$ for the Gunn effect) current oscillations.

Fig. 5 shows directly the shape and the propagation of these domains at different times, for two samples with a different contact geometry. The bar shaped samples with rectangular contacts clearly shows a domain which is perpendicular to the current propagation direction and which moves from the cathode to the anode. The structure formation is clearly very different from the filamentary structures discussed in connection with the breakdown in air. The sample exhibits a low electric field outside and a high electric field inside the domain. These two field values correspond to the stable points the $j-E$ curve in Fig. 1. The stable point at low electric field corresponds to a lower resistance state than the higher field point in the domain. This is demonstrated very directly in the sample with two circular electrodes in Fig. 5. The electrical domain is now circular shaped and retains this circular shape if it propagates away from the nucleating contact just before it arrives at the anode. This shape is very different from what would intuitively be expected from that of the dipolar electric field between the two electrodes. This behaviour occurs because the resistivity of the material outside the domain is much lower than that in the domain. Therefore, the potential due to current flow, drops almost entirely over the domain whereas the inside and outside regions are basically equi-potential planes, essentially at the cathode, respectively at the anode potential.

It is interesting to note that this topology is closely related to the details of the $j-E$ characteristic. Although knowledge of the $\mathrm{N}$-shape of this characteristic is enough to expect the formation of domains, the particular circular shape relies on a quantitative argument, namely that the conductivity in the low field region must be very much higher than that in the high field region.

\section{Summary}

We have shown two different examples of pattern formation in strongly non-linear conducting media, directly visualizing the formation of current filaments and of electrical domains for S- and $\mathrm{N}$-shaped $j-E$ characteristics, respectively. The pattern of the filamentary structure is shown to be influenced strongly by an external magnetic field. In particular, it can be shown that treating the patterns as a fractal structure the effect of the external magnetic field may be described by a continuous variation of the fractal dimension.

Pattern formation in non-linear systems is intensively studied in hydrodynamics, heat flow and chemical reactions. It is known to be essential in the understanding of non-linear current transport but because there the inherent temporal structure formation is mostly not directly visible, it is much less studied. The two examples given here demonstrate that also in dielectric breakdown these effects can be made visible and may provide an equally rich subject of study.

\section{References}

[1] M.C. Cross and P.C. Hohenberg, Rev. Mod. Phys. 65 (1993) 851.

[2] G. Ahlers, Physica D 51 (1991) 421 .

[3] E. Schöll, in: Handbook of Semiconductors, Vol. 1, ed. T.S. Moss (North-Holland, Amsterdam, revised ed., 1992) ch. 8 .

[4] G.Ch. Lichtenberg, Novi Comment Göttingen 8 (1778) 168.

[5] For a review of the basic physics of surface discharges see: A.M. Howatson, An Introduction to Gaseous Discharges (Pergamon, New York, 1976).

[6] S. Lazigaldie, J. Appl. Phys. 61 (1987) 90

[7] L. Niemeyer, L. Pietronero and H.J. Wiesmann, Phys. Rev. Lett. 52 (1984) 1033.

[8] B. Mandelbrot, The Fractal Geometry of Nature (Freeman, San Francisco, 1982).

[9] P. Uhlig, J.C. Maan and P. Wyder, Phys. Rev. Lett. 63 (1989) 1968.

[10] H.J. Wiesmann and L. Pietronero, in: Fractals in Physics (North-Holland, Amsterdam, 1986) p. 151.

[11] H.K. Sacks and A.G. Milnes, Int. J. Electronics 28 (1970) 565.

[12] S.M. Sze, Physics of Semiconductor Devices (Wiley, New York, 2nd ed., 1981). 